\title{
G6PD 缺乏症诊疗及其与地中海贫血关联发病研究新进展
}

\section{New progress in the diagnosis and treatment of G6PD deficiency and its association with thalassemia}

\author{
凌永嫦 李妹燕 王俊利通讯作者 \\ Yongchang Ling Meiyan Li Junli Wang \\ 右江民族医学院附属医院 中国・广西 百色 533000
}

Affiliated Hospital of Youjiang Medical College for Nationalities, Baise, Guang xi, 533000, China

\begin{abstract}
摘要: 葡萄糖一6一磷酸脱氢酶( glucose-6-phosphate dehydrogenase, G6 PD) 缺乏症属于 X 连锁不完全显性遗传病, 基因 突变是其主要发病原因。该疾病目前无特效治疗方法, 早期预防和诊断是我国优生优育工作的重点之一, 医务人员及时给予 患者合理的建议,让患者尽量避免使用有可能引起溶血的食物及药物, 并及时诊治该疾病引起的新生儿高胆红素血症, 是预 防和诊治该疾病的重点。G6PD 缺乏与地中海贫血是遗传上独立的溶血性疾病,但近年来研究者发现 G6PD 与地中海贫血发 病存在关联。因此, 本文对 G6PD 缺乏症的诊疗及与地中海贫血之间的关联发病进行了简要综述。
\end{abstract}

\begin{abstract}
Glucosate -6 - phosphate dehydrogenase (G6PD) deficiency is an X-linked incomplete dominant disease, and gene mutation is the main cause of the disease. At present there is no specific treatment, the disease prevention and early diagnosis is one of the focal point of prenatal and postnatal care and foster physical fitness among our country, and patients with medical staff in a timely manner to give reasonable Suggestions, let patients try to avoid using may cause hemolysis of food and drugs, and timely diagnosis and treatment the disease caused by high neonatal bilirubin hematic disease, is the key of the prevention and treatment of the disease. G6PD deficiency and thalassaemia are genetically independent hemolytic diseases, but in recent years, researchers have found that G6PD is associated with the incidence of thalassaemia. Therefore, this paper briefly reviewed the diagnosis and treatment of G6PD deficiency and its association with thalassemia.
\end{abstract}

关键词: G6PD 缺乏; 诊疗进展; 地中海贫血; 关联发病

Keywords : G6PD deficiency; Diagnosis and treatment progress; Thalassemia; Associated with the onset of 基金项目: 2018 年度广西重点研发项目(桂科 AB18221029)，2011 年度百色市科技攻关项目(20110507)

DOI : 10.36012 /pmr. v2i5. 2740

【作者简介】凌永嫦 $(1985 \sim)$, 女, 医学学士, 在读硕士研究生, 从事遗传病研究。

通讯作者: 王俊利, 博士/教授, 研究生导师, 从事不孕不育分子遗传学基础与临床研究。 
G6PD 缺乏症属于遗传性基因缺陷的疾病, 影响着全球 4 亿多人, 全球发病率为 $4.9 \%^{[1]}$ 。G $6 \mathrm{PD}$ 缺乏的许多患者可 终身不发病, 基本不影响其生活质量及寿命, 但当患者暴露 于溶血性诱发因素 (氧化性食物、药物、感染等) 可引起急性 溶血。目前尚无针对这种缺陷的特定治疗方法, 医学态度仅 限于预防溶血性危机的发生 (避免引发因素), 并在出现情况 时采取支持措施。该疾病在全球广泛分布, 在中国的广东、 广西、云南、海南、贵州、四川等地区发病率较高, 且男性的发 病率明显高于女性。

\section{G6PD 缺乏症的发病机制及遗传特征}

\subsection{G6PD 缺乏的发病机制}

G6PD 基因位于 X 染色体长臂 2 区 8 带 (Xq28), 能编码 515 个氨基酸。G6PD 缺乏是由 G6PD 的基因突变引起的遗 传性疾病, 其突变主要集中在第 4、5、6、8、10 号外显子 ${ }^{[2]}$, 可 引起 G6PD 转录产物合成障碍, 进而改变氨基酸序列。目 前,全球 G6PD 变异型约 400 多种, 并且在 G6PD 基因中总 共鉴定出 200 多个不同的点突变 ${ }^{[3-4]}$; 而在中国人群中鉴定 出至少 35 种点突变, c. 1376 G $>$ T、c. 1388 G > A、c. 95 A $>$ $\mathrm{G}$ 是中国人常见的致病基因型, 突变频率均>70\% ${ }^{[4-5]}$ 。舒 慧英等 ${ }^{[5]}$ 通过对成都市 12 名 G6PD 缺乏的患儿进行二代基 因测序, 检测出 1 种复合基因位点突变和 6 种单个基因位点 突变 (主要包括 c. $1376 \mathrm{G}>$ T、c. $487 \mathrm{G}>\mathrm{A}$ 和 c. $1388 \mathrm{G}>\mathrm{A}$ ), 与我国常见的 3 种突变类型有一定的差异。

\subsection{G6PD 缺乏的遗传特征}

G6PD 缺乏症的遗传突变基因由女性携带, 然而其致病 对象主要为男性 (G6PD 缺乏者中 $90 \%$ 是男性) ${ }^{[1]}$ 。按遗传 规律, 男性 G6PD 缺乏症患者的基因型仅存在半合子形式, 而女性多为杂合子形式。由于女性杂合子存在异质性, 基因 型也表现为多样性, 因此女性杂合子是比较特殊的群体 ${ }^{[5]}$, 部分女性患者可以有着和男性患者严重酶缺乏一样的临床 表现, 然而也存在部分女性患者无任何发病症状。李否等 [6]报道女性携带单一突变基因者, 酶活性有很大的变异性, 导致表型篮查检出率低,而携带双重突变者 G6PD 酶活性受
到严重影响, 临床表现同男性半合子。因此, 考虑可以从男 性后代基因型推测其母亲基因型或可能的基因型, 防止 漏检。

\section{G6PD 缺乏症的临床特征及诊断治疗}

\subsection{G6PD 缺乏的临床特征}

G6PD 缺乏症的临床表现多样, 大多数患者在稳定状态 下无临床表现或仅有轻微的症状, 当暴露于细菌、病毒感染、 亘豆、氧化性药物等诱发因素时, 可导致红细胞内血红蛋白 合成受损和不稳定的血红蛋白形成, 可出现急性的溶血反 应。药物性溶血、亘豆病、感染性溶血、先天性非球形细胞溶 血性贫血及新生儿黄疸是是其常见的临床分型。G6PD 催 化磷酸戊糖途径所产生的还原型辅酶 II (NADPH) 能保持谷 胱甘肽还原酶的还原形式, 从而维持红细胞膜稳定性 ${ }^{[7]}$, 因 此 G6PD 对于维护健康机体的 NADPH 和氧化还原稳态至 关重要。G6PD 对磷酸化高度敏感, 是导致酶活性丧失引起 溶血性贫血的主要原因之一 ${ }^{[8]}$ 。大量的研究结果表明, G6PD 与血液相关的疾病有关, 主要是 G6PD 缺乏引起的溶 血性贫血。急性溶血性贫血是 G6PD 缺乏症最常见的临床 表现, 发生在红细胞处于氧化应激状态时, 通常由感染, 氧化 剂或食物引起。亘豆是 G6PD 缺乏症患者的急性诱发因素, 可导致严重的急性溶血 ${ }^{[9]}$ 。溶血的严重程度可能受许多因 素影响, 包括同时给药, 肝功能和年龄。除上述的急性发病 外,G6PD 缺乏所致的溶血一般呈慢性经过, 并可造成相对 罕见的先天性非球形红细胞溶血性贫血 ${ }^{[2-10]}$ 。

\subsection{G6PD 缺乏的诊断}

G6PD 缺乏症的诊断可以通过多种方法进行。首先可 以测量酶活性水平以确定患者的 G6PD 表达; 另外, 分子分 析可以用于检测编码 G6PD 的基因的突变。目前,临床上最 常用的是 G6PD 酶活性检测, 其中, G6PD/6PGD 比值法作为 一种操作简便的临床检测技术, 具有特异性高、结果可靠等 优点, 能明显提高 G6PD 缺乏患者检测结果的准确性 ${ }^{[11]}$ 。 但是女性杂合子酶活性有较大的异质性, 上述酶法测定存在 很大局限性, G6 PD 缺乏常常不能被检测出来。出于临床原 
因需要精确诊断时, 应考虑分子分析, 但分子分析可能无法 检测到所有变体及反映酶在所有条件下的功能活性, 且网状 细胞浓度和红细胞寿命也会影响酶的功能活性。因为大多 数的 G6PD 缺乏为点突变, 首先可选用聚合酶链反应一限制 性片段长度多态性直接进行突变分析, 如果此种方法为阴性 结果, 或者没有明确的常见突变, 则可以进行直接的 Sanger 测序; 此方法可检测出不常见的突变以及新突变 ${ }^{[12]}$ 。由于 Sanger 测序效率的提高以及 G6PD 缺乏症中基因突变的显 著异质性, 直接进行 Sanger 测序已成为一种更广泛使用的 方法 ${ }^{[13]}$, 并且该序列分析的阳性结果可以视为独立的诊断 结果。但是, 如果未发现突变, 则只有定量分析才能确定 G6PD 缺乏症。下一代测序 (NGS) 在血液系统疾病的诊断 和预后治疗方面具有具有较高的可靠性, 能检测出基因的新 突变以及多态性, 并能准确检测基因的已知的突 ${ }^{\text {变 }}{ }^{[12]}$ 。 $\mathrm{NGS}$ 的新进展使许多与红细胞疾病有关的基因同时测序成 为可能, 在病因未知的溶血情况下, 所有与红细胞酶缺乏症 相关的潜在基因都可以通过单个分子测定法进行检查, 而非 几个分子和生化测定法进行检测, 这对 G6PD 缺乏症诊断具 有重要的价值 ${ }^{[14]}$ 。

\subsection{G6PD 缺乏的治疗}

G6PD 缺乏的治疗以预防为主, 严重者采用对症治疗, 目前仍无法治愈。在 G6PD 缺乏症的高发地区, 所有新生儿 应进行 G6PD 缺乏症篮查, 并给予患儿的家长进行健康教 育, 禁用、慎用某些食物及药物 ${ }^{[15]}$ 。当出现急性溶血时, 应 采取相应的治疗措施, 并停止摄人可疑食物或药物; 严重贫 血时, 可通过输血以纠正贫血, 并且要避免肾衰竭的发生。 对于 G6PD 缺乏伴新生儿高胆红素血症, 应根据胆红素的水 平给予蓝光照射或换血疗法, 同时预防胆红素脑病的发 生 ${ }^{[16]}$ 。从 G6PD 缺乏的发病本质考虑, 基因治疗可能为其 有效的治疗方法。周燕霞等 ${ }^{[16]}$ 报道可将 G6PD 缺乏患者的 体细胞重编程为多能干细胞, 然后利用基因编辑技术 $\mathrm{CRISPR} / \mathrm{Cas} 9$ 修复多能干细胞中的致病突变, 再将修复后 的多能干细胞诱导分化为红系祖细胞移植到 G6PD 缺乏患 者体内, 从而达到治疗的目的。除此之外, 该技术还能纠正
其他多种严重的遗传疾病 (如地中海贫血、镰状红细胞贫血. 等), 是具有广阔的应用前景的新型遗传性疾病治疗方法。

\section{G6PD 缺乏症与地中海贫血发病关联}

地中海贫血是由一条或多条正常的珠蛋白肽链合成受损, 其 异常合成可导致无效红细胞生成、溶血, 并可出现不同程度 的慢性溶血性贫 血 $^{[17]}$ 。我国地中海贫血以南方多见, 并根 据缺失或减少珠蛋白基因进行分型, 主要以 $\alpha$ 型和 $\beta$ 型为 主。其中, $\alpha$ 地中海贫血根据突变基因又可分为缺失和非缺 失两种类型, 且严重程度与其基因型有关 ${ }^{[18]}$ 。目前地中海 贫血仍是不可治愈的, 轻型地中海贫血不需治疗, 但中、重度 型贫血需进行频繁输血、去铁等治疗, 给其家庭带来沉重的 经济负担和精神压力, 造成社会医疗负担, 同时也影响国家 的人口质量。地中海贫血也是我国优生优育工作的重点之 一, 应加强对该疾病的了解及预防意识, 避免中、重度地贫患 儿的出生。虽然地中海贫血和 G6PD 缺乏症是相对独立的 遗传性溶血性疾病, 但两者又存在一定的关联性, 在普遍存 在的 G6PD 缺乏症人群中, 血红蛋白 $\mathrm{S}$ 和地中海贫血并存的 频率很高。据报道, G6PD 缺乏可能与其他遗传修饰 (如地 中海贫血、血红蛋白 $\mathrm{f}$ 水平和镰状红细胞贫血的单倍型) 相 互作用有关 ${ }^{[19]}$ 。贫血、肿瘤或感染等多种因素可能会使 G6PD 基因发生改变, 并促进其表达。地中海贫血患者体内 发生溶血现象时会刺激机体生成大量的新生红细胞,而红细 胞的寿命又依赖于 G6PD 酶, 因此新生的红细胞内 G6PD 活 性相对较高。除此之外,地中海贫血和 G6PD 缺乏症还都存 在遗传多样性。国内报道 ${ }^{[20]}$, 新疆哈萨克族和维吾尔族人 群的 G6PD 基因和 $\beta$ 珠蛋白基因不但含有中东地区和地中 海地区特有的变异型, G6 PD 缺乏还含有中国常见的基因型 c. $1376 \mathrm{G}>\mathrm{T}$, c. $1388 \mathrm{G}>\mathrm{A}$ 和 c. $95 \mathrm{~A}>\mathrm{G}$ 。 $\mathrm{Wu}$ 等 $^{[21]}$ 报道: 梅 州市 8 个县地中海贫血和 G6PD 突变分布均存在一定差异, 该地区 G6PD 缺乏患者的主要突变类型包括: c. $95 \mathrm{~A}>\mathrm{G}, \mathrm{c}$. $1388 \mathrm{G}>\mathrm{A}$ 和 c. $1376 \mathrm{G}>\mathrm{T}$ 。以上研究表明不同地区和种 族人群中地中海贫血和 G6PD 缺乏基因突变发生频率和类 型可不相同。相关研究报道, G6 PD 缺乏与地中海贫血珠蛋 白的复合基因突变会有不同的临床表型, 与单纯的地中海贫 
血遗传相比, $\mathrm{Hb}$ 水平, 血红蛋白比容, 平均红细胞体积和平 均红细胞量显示出更低的平均值, 导致红细胞病变恶化; 而 $\beta$ 地贫患者体内新生红细胞的 G6PD 酶活性相对较高 ${ }^{[22-24]}$ 。 陈嵘等 ${ }^{[25]}$ 对广州地区的 G6PD 缺乏症患者研究发现: 不管 是否合并地贫, 男性 G6PD 缺乏患者比女性患者酶活性明显 降低; 而 G6PD 缺乏合并地贫的患者比单纯 G6PD 缺乏患者 的酶活性高。胡楚霞等 ${ }^{[26]}$ 对 459 例地贫育龄妇女检测改良 红细胞 G6PD 定量比值, 结果表明改良红细胞 G6PD 定量比 值增高与 $\alpha$ 地中海贫血、 $\beta$ 地中海贫血基因存在相关性, 且地 贫基因缺陷度或临床症状严重程度与 G6PD 活性成正相关。 因此, 把 G6PD 活性、改良红细胞 G6PD 定量比值、MCV、 $\mathrm{MCH}$ 等结合应用于地中海贫血篮查, 对地贫诊断具有一定 的指导意义。但 G6PD 缺乏合并地中海贫血的患者, 尤其是 女性杂合子, 应进行基因检测, 必要时行家系分析, 以防 漏检。

\section{4 展望}

基于 G6PD 的溶血性贫血影响全球超过 5 亿人; 且 G6PD 是 一种典型的维持生长发育的管家酶, 减少 G6PD 活性可阻止 正常细胞增殖以及胚胎和机体的发育 ${ }^{[27]}$ 。目前许多研究发 现 G6PD 不但与细胞生理有关, 而且在某些疾病发生发展中 发挥着至关重要的作用。当 G6PD 缺乏合并其他疾病(如疮 疾、糖尿病、地中海贫血. 时, 患者基因突变也在变化,临床确 诊一定程度上受到了限制。近年来关于 G6PD 缺乏与地中 海贫血的关联及其作为诊断标志物的潜力可用文献尚有限。 同时, 关于 G6PD 缺乏症的基因组调控及表观遗传学层面的 研究也少见。G6PD 缺乏目前仍无法被治愈, 是一个全球都 关注的健康问题。因此, 开发 G6PD 缺乏的诊断工具表达和 酶谱分析至关重要; 同时探索该疾病的基因治疗以及与其他 疾病的关联性可能成为未来该领域探究的又一热点。希望 随着研究的进一步深人, G6PD 与相关疾病关联性发病的机 制和治疗方法也有望进一步被揭示。

\section{参考文献}

[1] DePina, A. J. ; Pires, C. M. ; Andrade, A. J. B. ; Dia, A. K. ; Moreira, A. L. ; Ferreira, M. C. M. ; Correia, A. J. ; Faye, 6
O. ; Seck, I. ; Niang, E. H. A. The prevalence of g lucose-6 - phosphate dehydrogenase defificiency in the Cape Verdean population in the context of malaria elimination. PLoS ONE 2020,15, e0229574.

[2] MINUCCI A, MORADKHANI K, HWANG M J, et al. Glucose $-6-$ phosphate dehydrogenase (G6PD) mutations data base:review of the"old"and update of the new mutations $[\mathrm{J}]$. Blood Cells Mol Dis, 2012, 48 (3) : 154-165.

[3] Gómez-Manzo S, Marcial-Quino J, Vanoye-Carlo A, et al. Glucose $-6-$ Phosphate Dehydrogenase: Update and Analysis of New Mutations around the World. [J]. Int J Mol Sci. 2016, 17(12): e2069.

［4］林芬, 杨辉, 杨立业. 我国葡萄糖一 6 一磷酸脱氢酶缺乏症的分 布特征和基因突变 $[\mathrm{J}]$. 分子诊断与治疗杂志，2016，8(02)： $73-77$.

[5] 舒慧英, 张庆,李蕙, 等. 葡萄糖 -6 - 磷酸脱氢酶缺乏症基因 突变分析 $[\mathrm{J}]$. 中华妇幼临床医学杂志 (电子版), 2018, 14 (03) : $291-295$.

［6］李磊, 郭洪创, 肖奇志, 等. 逆遗传方向研究男性 G6PD 缺乏症 及其母亲的表型与基因型 $[\mathrm{J}]$. 中国生育健康杂志，2019，30 (03): $232-236$.

[7] Luzzatto, L. ; Nannelli, C. ; Notaro, R. Glucose-6-phosphate dehydrogenase defificiency. Hematol. Oncol. Clin. N. Am. 2016, 30, 373 $-393$

[8] Stanton RC. Glucose-6-Phosphate Dehydrogenase, NAD PH, and Cell Survival. IUBMB Life. 2012;64(5):362 - 369

[9] Luzzatto L, Arese P. Favism and glucose $-6-$ phosphate dehydrogenase deficiency. N Engl J Med. 2018; 378: 1068 $-1069$.

[10 ] Belfield KD, TichyEM. Review and drug therapy implicationsof glucose $-6-$ phosphate dehydrogenase deficiency $[\mathrm{J}]$. Am J Health Syst Pharm, 2018, 75(3): 97-104.

[11] Jiang J, Li B, Cao W, et al. Screening and prevention of neonatal glucose $-6-$ phosphate dehydrogenase deficiency in Guangzhou, China[J]. Genet Mol Res, 2014, 13(2) : 42724279. 
[12] D Roper, Layton M, Rees D, et al. Laboratory diagnosis of G6PD deficiency. A British Society for Haematology Guideline $[J]$. Br J Haematol, 2020, 189(1): 24-38.

[13] A Minucci, Moradkhani K, Hwang M-J, et al. Glucose-6 - phosphate dehydrogenase (G6PD) mutations database: review of the "old" and update of the new mutations $[\mathrm{J}]$. Blood Cells Mol Dis, 2012, 48(3): 154-165.

[14] Bogari NM. Next generationsequencing (NGS) in glucose -6 - phosphate dehydrogenase (G6PD) deficiency studies $[\mathrm{J}]$. Bioinformation, 2016,12(2):41-43.

[15] SKÖLD M B, SVENDSEN R P, PEDERSEN E B. Favism after ingestion of fava beans in a three - year - old child with glucose $-6-$ phosphate dehydrogenase deficiency[J]. Ugeskr Laeger, 2017, 179 (20) : V01170004.

[16 ] 周燕霞, 张鹏辉. 基于 CRISPR/Cas9 技术治疗 G6PD 缺乏症 的运用前景 $[\mathrm{J}]$. 重庆医学, 2019, 48(03): 473-475.

[17] Jr MH, Campbell J. Alpha and beta thalassemia. American Family Physician[J]. American Family Physician, 2009, 80 (4) $: 339-344$.

[18] SARKAR S K, SHAH M S, BEGUM M, et al. Red Cell Alloantibodies in Thalassaemia Patients Who Received Ten or More Units of Transfusion. [J]. Mymensingh medical journal :MMJ ,2019,28(2):364.

[19] Matthew - S Karafin, Fu Xiaoyun, Alessandro Angelo-D, et al. The clinical impact of glucose-6- phosphate dehydrogenase deficiency in patients with sickle cell disease[J]. Current Opinion in Hematology, 2018, 25(6).

[20］韩璐好. 新疆少数民族 G6PD 缺乏症及地中海贫血分子遗传
学研究 $[D]$. 中山大学, 2016 .

[21] Wu H, Zhu Q, Zhong H, et al. Analysis of genotype distribution of thalassemia and G6PD deficiency among Hakka popu lation in Meizhou city of Guangdong Province[J]. J Clin Lab Anal,2019,e23140.

[22] Pengon, J ; Svasti, S; Kamchonwongpaisan, S; et al. Hematological parameters and red blood cell morphological abnormality of Glucose-6-Phosphate dehydrogenase deficiency co - inherited with thalassemia. [J]. Hematol Oncol Stem Cell Ther. $2018,11(1): 18-24$

[23] Chang, A K ; Ginter Summarell, C C; Birdie, P T; et al. Genetic modifiers of severity in sickle cell disease $[\mathrm{J}]$. Clin $\mathrm{He}^{-}$ morheol Microcirc. 2018,68(2-3):147-164.

[24] Ouattara, AK; Yameogo, P; Traore, L; et al. Prevalence, genetic variants and clinical implicatio7ns of $\mathrm{G}-6-\mathrm{PD}$ deficiency in Burkina Faso: a systematic review. [J]. BMC Med Genet. $2017,18(1): 139$

[25］陈嵘,陈桂兰, 屈艳霞, 等. G6PD 缺乏症合并地中海贫血患者 G6PD 活性和基因突变类型分析 $[\mathrm{J}]$. 中国优生与遗传杂志, $2015,23(06): 26-27$.

[26] 胡楚霞, 袁明生. 不同类型地中海贫血基因的改良红细胞 G6PD 定量比值的统计分析与意义 $[\mathrm{J}]$. 解放军预防医学杂 志, 2020, 38(02): 81-83.

[27] Wu, Y. H. ; Lee, Y. H. ; Shih, H. Y. ; Chen, S. H. ; Cheng, Y. C. ; Tsun-Yee Chiu, D. Glucose-6- phosphate dehydrogenase is indispensable in embryonic development by modulation of epithelial - mesenchymal transition via the NOX/Smad3/miR-200b axis. Cell Death Dis. 2018, 9, 10. 